(с) Н.О. Ховасова ${ }^{1,2 *}$, А.В. Наумов ${ }^{1,2}$, О.Н. Ткачева ${ }^{1}$, Е.Н. Дудинская ${ }^{1,3}$, В.И. Мороз ${ }^{1,2}$

'Кафедра болезней старения ФДПО РНИМУ им. Пирогова, Москва

²Лаборатория заболеваний костно-мышечной системы, РГНКЦ, Москва

Злаборатория возрастных метаболических и эндокринных нарушений, РГНКЦ, Москва

Обоснование. Падения в пожилом возрасте - многофакторный синдром. Одним из модифицируемых факторов является полипрагмазия. Для обоснованной коррекции полипрагмазии в гериатрии используются STOPP/START-критерии. Цель. Оценка распространенности полипрагмазии, анализ и коррекция фармакотерапии с помощью STOPP/ START-критериев у пациентов с падениями.

Материалы и методы. В исследование включены 655 госпитализированных в гериатрическое отделение пациен-

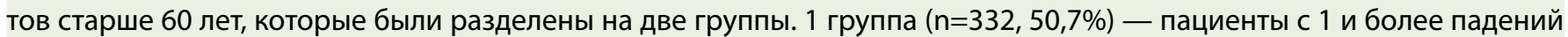
за год, 2 группа $(n=323,49,3 \%)$ - пациенты без падений. Выполнен анализ получаемой терапии до госпитализации. После чего на основании показаний, противопоказаний, STOPP/START-критериев у пациентов с синдромом падений проводилась коррекция лекарственной терапии.

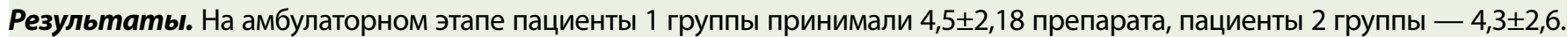
Полипрагмазия диагностирована у 150 (45,2\%) пациентов с падениями и у 122 (37,8\%) пациентов без падений. Пациенты с падениями достоверно чаще $(p<0,05)$ получали снотворные препараты, нестероидные противовоспалительные препараты (НПВП). Однофакторный анализ продемонстрировал, что падения ассоциированы с приемом НПВП (отношение шансов (ОШ) 2,15; 95\% доверительный интервал (ДИ) 1,38-3,35; p=0,001) и снотворных препаратов (ОШ 2,03; 95\% ДИ 1,02-4,02; p=0,047). У пациентов с падениями проведены аудит и коррекция лекарственной терапии: у 108 (32,5\%) пациентов количество назначенных препаратов было уменьшено, у 165 (49,7\%) - увеличено, а у 59 (17,8\%) - не изменилось. Пациентам с падениями чаще были назначены статины, противодементные препараты, антиконвульсанты и антидепрессанты как компоненты обезболивающей терапии при хроническом болевом синдроме, препараты хондроитина сульфата и глюкозамина сульфата для лечения остеоартрита, препараты кальция и антирезорбтивная терапия у пациентов с остеопорозом, противоанемические препараты. Всем пациентам был назначен витамин D. Реже назначались антиагреганты, дигоксин, снотворные препараты, ноотропы, НПВП. Отдельно были проанализированы STOPP/START-критерии и их частота у пациентов с синдромом падений. Всего выявлен 141 случай потенциально не рекомендованных, но назначенных лекарственных препаратов. Наиболее часто STOPР-критерии касались назначения НПВП ( $=53,37,6 \%)$ и ацетилсалициловой кислоты

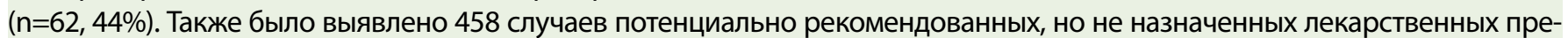
паратов. Наиболее часто START-критерии касались неназначения витамина D и статинов.

Заключение. Почти половина пожилых пациентов с синдромом падений имеют полипрагмазию. Эти пациенты чаще принимают снотворные препараты и НПВП. При аудите догоспитальной лекарственной терапии STOPP-критерии наиболее часто касались назначения НПВП и ацетилсалициловой кислоты, а START-критерии выявили отсутствие назначения витамина D и статинов.

КЛЮЧЕВЫЕ СЛОВА: падения; полипрагмазия; гериатрический синдром; STOPP/START-критерии.

\title{
POLYPHARMACY IN ELDERLY PATIENTS WITH FALLS
}

(c) Natalia O. Khovasova ${ }^{1,2 *}$, Anton V. Naumov ${ }^{1,2}$, Olga N. Tkacheva' ${ }^{1}$ Ekaterina N. Dudinskaya1,3, Victoriya I. Moroz ${ }^{1,2}$

'Department of diseases of aging of The Pirogov Russian National Research Medical University

${ }^{2}$ Laboratory of the Musculoskeletal System Diseases, Russian Gerontology Clinical Research Center ${ }^{3}$ Laboratory of Age-related Metabolic Endocrine Disorders Russian Gerontology Clinical Research Center

Background: Falls in elderly-a multifactorial syndrome. One of the modifiable factors is polypharmacy. STOPP/START criteria are used for correction of polypharmacy in geriatrics.

Aim: Assessment of the prevalence of polypharmacy, analysis and correction of pharmacotherapy using STOPP/START criteria in patients with falls.

Materials and methods: The study included 655 patients hospitalized in the geriatric department over 60 years of age, who were divided into two groups. Group $1(n=332,50.7 \%)$-patients with 1 or more falls, group 2 ( $n=323,49.3 \%)-p a t i e n t s$ without falls. The analysis of the received therapy before hospitalization was performed. After that, based on the indications, contraindications and STOPP/START criteria, drug therapy was corrected in patients with falls.

Results: Patients of group 1 took 4.5 \pm 2.18 drugs, group 2-4.3 \pm 2.6 . Polypharmacy was diagnosed in 150 (45.2\%) patients with falls and in $122(37.8 \%)$ patients without falls. Patients with falls were more likely to receive sleeping pills, NSAIDs. Univariate 
analysis showed that falls were associated with NSAIDs (OR 2.15, 95\% Cl 1.38-3.35, $\mathrm{p}=0.001$ ) and sleeping pills (OR 2.03, 95\% $\mathrm{Cl} 1.02-4.02, \mathrm{p}=0.047)$. An audit and correction of therapy was performed: in $108(32.5 \%)$ patients the number of prescribed drugs was reduced. Patients with falls were prescribed statins, antidementia drugs, anticonvulsants and antidepressants as components of therapy for chronic pain syndrome, chondroitin sulfate and glucosamine sulfate for the treatment of osteoarthritis, calcium and antiresorbtive therapy, antianemic drugs, vitamin D. Antiplatelet agents, digoxin, sleeping pills and NSAIDs were less frequently prescribed. STOPP/START criteria and their frequency in patients with falls were analyzed. 141 cases of potentially non-recommended but prescribed medications were identified. STOPP criteria were for the administration of NSAIDs $(n=53,37.6 \%)$ and acetylsalicylic acid $(n=62,44 \%)$. There were 458 cases of potentially recommended but not prescribed medications. The most common START criteria were not for the administration of vitamin D and statins. Conclusion. Half of elderly patients with falls have polypharmacy. These patients are more likely to take sleeping pills and NSAIDs. STOPP criteria most often concerned the appointment of NSAIDs and acetylsalicylic acid, and the START criteria revealed the absence of the appointment of vitamin $\mathrm{D}$ and statins.

KEYWORDS: falls; polypharmacy; geriatric syndrome; STOPP/START criteria.

\section{ОБОСНОВАНИЕ}

Падения в пожилом возрасте - многофакторный синдром, который складывается из сложного взаимодействия биологических, поведенческих, средовых и социально-экономических факторов [1]. Все эти факторы можно разделить на немодифицируемые (например, пожилой возраст, пол, деменция) и модифицируемые (например, ортостатическая гипотония, дефицит витамина D, анемия). Одним из серьезных модифицируемых факторов риска падений является полипрагмазия - одновременное назначение большого количества лекарственных средств. Наиболее часто речь идет о назначении 5 и более лекарственных препаратов [2, 3]. При этом чем большее количество лекарственных средств принимает пациент, тем выше риск падений [4].

Полипрагмазия, помимо повышения риска падений и переломов, ассоциирована с такими гериатрическими синдромами, как депрессия, снижение физического функционирования, когнитивные нарушения, делирий $[5,6]$. Хорошо известно, что полипрагмазия приводит к увеличению частоты лекарственных взаимодействий: чем больше количество принимаемых препаратов, тем чаще развиваются лекарственные взаимодействия [7]. Кроме того, данные исследований последних лет демонстрируют повышение риска смерти от любых причин у пациентов с полипрагмазией: прием 5 лекарственных препаратов ассоциирован с повышением риска смерти в 1,31 раза, а прием 10 и более лекарственных препаратов - в 1,96 раза [8].

Очевидно, что борьба с полипрагмазией является приоритетной задачей любого клинициста, а для гериатра - еще и одним из способов профилактики падений и переломов. Сегодня в арсенале врача-гериатра есть инструмент для обоснованной коррекции полипрагмазии - STOPP/START-критерии, разработанные в 2008 г. в Ирландии. STOРР-критерии - лекарственные препараты, которые не рекомендуется применять у пожилых людей, a START-критерии - лекарственные препараты, которые рекомендованы к назначению пожилым пациентам $[9,10]$.

\section{ЦЕЛЬ ИССЛЕДОВАНИЯ}

Целью нашей работы было оценить распространенность полипрагмазии, проанализировать и провести коррекцию фармакотерапии с помощью STOPP/ START-критериев у пациентов с синдромом падений.

\section{МАТЕРИАЛЫ И МЕТОДЫ}

Место и время проведения исследования

Исследование проводилось на базе Российского геронтологического научно-клинического центра города Москвы в период июнь 2019-февраль 2021 г.

\section{Изучаемые популяции}

Пациенты (n=655) старше 60 лет, госпитализированные в гериатрическое отделение. Критерии включения: возраст 60 лет и старше; согласие на участие в исследовании. Критерии исключения: возраст менее 60 лет; отказ от участия в исследовании.

Способ формирования выборки из изучаемой популяции: сплошной.

\section{Дизайн исследования}

Одноцентровое одномоментное наблюдательное проспективное исследование.

В зависимости от факта падения в течение последнего года пациенты были разделены на две группы. 1 группа (332 человек, 50,7\%) - пациенты с эпизодом одного и более падений за год, 2 группа (323 человек, 49,3\%) пациенты без падений. Пациенты обеих групп были сопоставимы по полу, возрасту, семейному и социальному статусам. Также группы были сопоставимы по коморбидной патологии, распространенность которой оказалась значительной: в среднем на одного пациента приходилось 5 заболеваний (табл. 1).

\section{Методы}

У всех пациентов проводился анализ получаемой фармакотерапии до госпитализации, после чего на основании показаний, противопоказаний, STOPP/START-критериев у пациентов с синдромом падений проводилась коррекция лекарственной терапии. Полипрагмазией считался регулярный прием 5 и более лекарственных препаратов.

\section{Статистический анализ}

База данных создана в программе Microsoft Excel 2020 для MacOS. Количественные показатели приведены в виде среднего арифметического (М) с соответствующим стандартным отклонением (SD). Качественные данные представлены в виде абсолютных чисел и относительных частот. Нормальность распределения 
Таблица 1. Характеристика пациентов, включенных в исследование

\begin{tabular}{|c|c|c|c|c|}
\hline Характеристика & $\begin{array}{c}\text { Пациенты } \\
\text { с падениями } \\
(\mathbf{n}=332)\end{array}$ & $\%$ & $\begin{array}{c}\text { Пациенты без } \\
\text { падений } \\
\text { (n=323) }\end{array}$ & $\%$ \\
\hline Женщины & 264 & 79,5 & 267 & 82,6 \\
\hline Возраст, лет & $74,9 \pm 7,7$ & & $74,1 \pm 8,2$ & \\
\hline Мужчины & 68 & 20,5 & 56 & 17,3 \\
\hline Возраст, лет & $78,1 \pm 8,2$ & & $76,6 \pm 9,4$ & \\
\hline Наличие инвалидности & 151 & 45,5 & 127 & 39,3 \\
\hline \multicolumn{5}{|l|}{ Семейный статус } \\
\hline Женат/замужем & 126 & 38 & 107 & 33,1 \\
\hline В разводе & 31 & 9,3 & 36 & 11,1 \\
\hline Вдовец/вдова & 161 & 48,5 & 166 & 51,4 \\
\hline Не женат/не замужняя & 14 & 4,2 & 14 & 4,4 \\
\hline \multicolumn{5}{|l|}{ Проживание } \\
\hline В семье & 120 & 36,2 & 142 & 44 \\
\hline Одинокое & 203 & 61,1 & 174 & 53,9 \\
\hline В пансионате & 9 & 2,7 & 7 & 2,1 \\
\hline Индекс Чарльсон & $5,7 \pm 2,2$ & & $5,7 \pm 2,1$ & \\
\hline Количество заболеваний у одного пациента & $5,3 \pm 2,2$ & & $5,5 \pm 2,1$ & \\
\hline
\end{tabular}

Примечание: $p>0,05$ во всех случаях.

полученных параметров оценивалась при помощи критериев Шапиро-Уилка. Для ненормально распределенных показателей применялся непараметрический критерий Манна-Уитни, для нормально распределенных - Т-критерий Стьюдента. Различия считали значимыми при $p<0,05$. Взаимосвязи между переменными оценивали при помощи однофакторного анализа, для чего использовали бинарную логистическую регрессию с вычислением отношения шансов (ОШ) и 95\% доверительного интервала (ДИ). Различия считали значимыми при $\mathrm{p}<0,05$.

\section{Этическая экспертиза}

Протокол исследования рассмотрен и одобрен локальным этическим комитетом ФГБОУ ВО РНИМУ им. Н.И. Пирогова ОСП РГНКЦ (протокол заседания № 25 от 17.06.2019).

\section{РЕЗУЛЬТАТЫ}

Среди 655 включенных в исследование пациентов падения в течение последнего года перенесли 332 (50,7\%) человека. Они составили 1 группу наблюдения. У 323 (49,3\%) пациентов падений не было, эти пациенты вошли во 2 группу наблюдения.
Учитывая высокую коморбидную отягощенность, было ожидаемо, что пациенты получали большое количество лекарственных препаратов. Так, на амбулаторном этапе пациенты 1 группы принимали от 0 до 13 препаратов, медиана составила 4 (3; 6); пациенты 2 группы от 1 до 12 препаратов, медиана - 4 (3; 6), p=0,402. Данные представлены как Ме (25\%; 75\%).

Частота приема различного количества лекарственных препаратов представлена на рис. 1. Как видно из рис. 1, полипрагмазия была диагностирована у 150 (45,2\%) пациентов с падениями и у 122 (37,8\%) пациентов без падений. При этом наблюдалась закономерность: среди пациентов, принимавших три и более препарата, упавших было больше $(64,8 \%$ и $54,8 \%, p=0,01), 4$ и более препарата - 77,5\% и 65,6\%, соответственно ( $p=0,001)$.

Структура фармакотерапии на догоспитальном этапе представлена в табл. 2. Согласно полученным данным, пациенты с эпизодами падений достоверно чаще ( $<<0,05)$ получали снотворные препараты, НПВП и ингибиторы протонной помпы. Чаще этим пациентам были назначены противопаркинсонические препараты, ноотропы, гормоны щитовидной железы. При помощи однофакторного дисперсионного анализа была изучена взаимосвязь между падениями и приемом лекарственных препаратов, указанных в табл. 2. Оказалось, что падения 


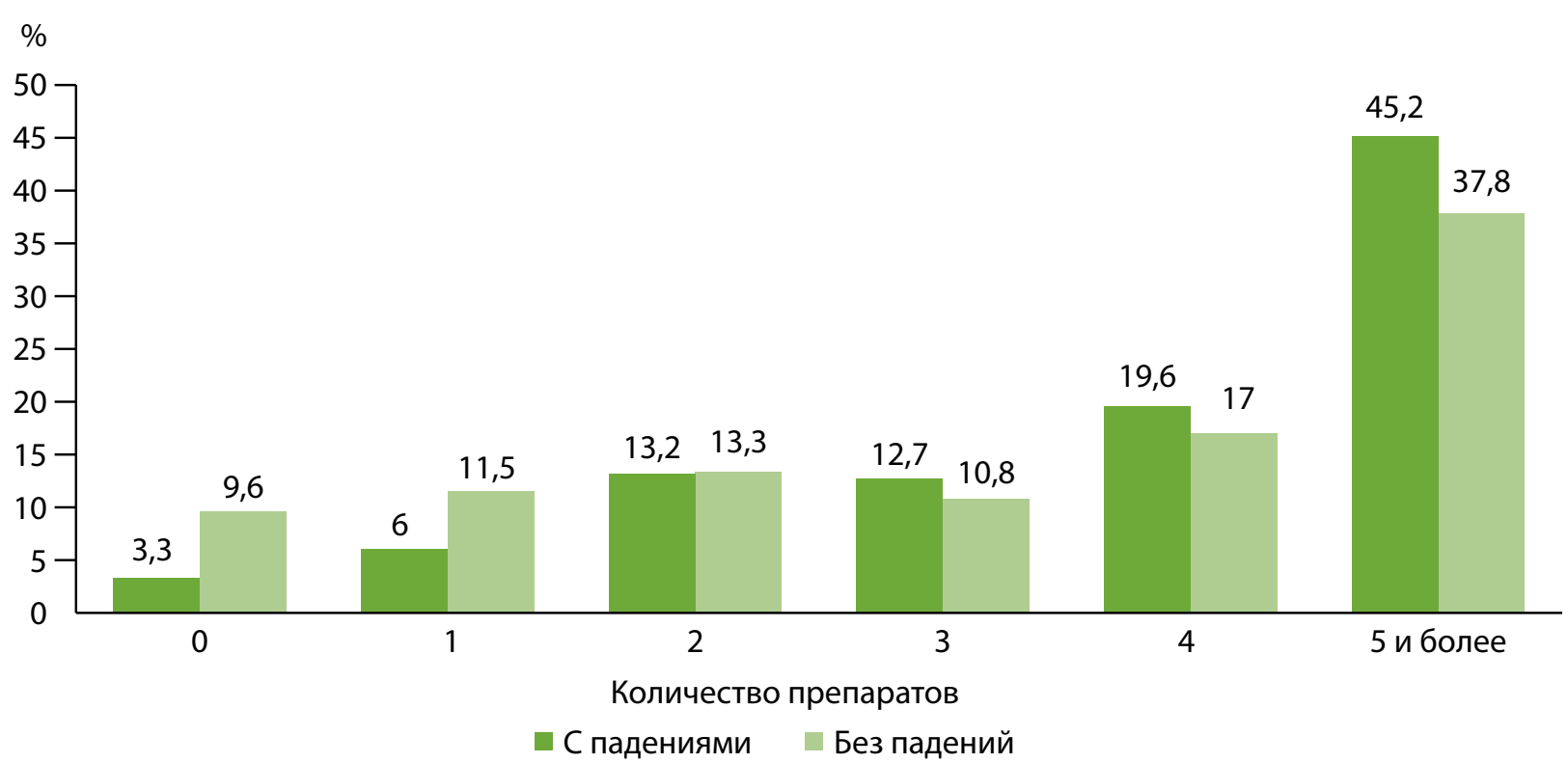

Рисунок 1. Количество принимаемых пациентами препаратов

ассоциированы с приемом НПВП (ОШ 2,15; 95\% ДИ 1,38-3,35; $p=0,001$ ) и снотворных препаратов (ОШ 2,03; $95 \%$ ДИ 1,02-4,02; $p=0,047)$. Ассоциаций не выявлено между падениями и приемом остальных лекарственных препаратов, указанных в табл. 2.

У пациентов 1 группы проведен анализ лекарственной терапии, после чего, учитывая показания, противопоказания и STOPP/START-критерии, она была скорректирована. В результате такой коррекции у 108 (32,5\%) пациентов количество назначенных препаратов было уменьшено, у 165 (49,7\%) - увеличено, а у $59(17,8 \%)$ пациентов - не изменилось. Однако отсутствие изменений общего количества препаратов не означало, что не изменились наименования назначенных препаратов (табл. 3).

Таким образом, пациентам с синдромом падений в стационаре достоверно чаще $(p<0,05)$ были назначены статины, противодементные препараты, антиконвульсанты и антидепрессанты как компоненты обезболивающей терапии у пациентов с хроническим болевым синдромом с нейропатическим и психогенным компонентами, препараты хондроитина сульфата и глюкозамина сульфата для лечения остеоартрита. Также чаще назначались препараты кальция и антирезорбтивная терапия у пациентов с остеопорозом. Всем пациентам был назначен витамин D. Противоанемические препараты - препараты железа и фолиевая кислота были назначены в 4 и 2,5 раза чаще, чем на амбулаторном этапе. Наоборот, достоверно реже $(p<0,05)$ были назначены антиагреганты, дигоксин, снотворные препараты, ноотропы, НПВП. Такие препараты, как валидол и корвалол, были отменены у всех пациентов, получавших их на догоспитальном этапе.

Отдельно были проанализированы STOPP/START-критерии (табл. 4 и 5) и их частота у пациентов с синдромом падений. Всего был выявлен 141 случай потенциально не рекомендованных, но назначенных лекарственных препаратов (STOPP-критерии) этим пациентам. Как видно из табл. 4, наиболее часто STOPР-критерии касались назначения НПВП ( $\mathrm{n}=53,37,6 \%)$ и ацетилсалициловой кислоты $(n=62,44 \%)$.
Также было выявлено 458 случаев потенциально рекомендованных, но не назначенных лекарственных препаратов (START-критерии). Как видно из табл. 5, наиболее часто START-критерии касались неназначения витамина D и статинов.

\section{ОБСУЖДЕНИЕ}

\section{Репрезентативность выборок.}

Набор участников исследования проводился только в федеральном научном центре - РГНКЦ. В исследование вошли госпитализированные в гериатрическое отделение пациенты.

\section{Сопоставление с другими публикациями}

Распространенность падений среди пожилых людей составляет 28-35\% в возрасте 65-70 лет и 32-42\% - старше 70 лет [11]. Еще более высокая частота падений наблюдается среди пожилых жителей домов престарелых [12]. Наши данные демонстрируют несколько более высокую частоту падений $(50,7 \%)$, чем в общей популяции, что может быть объяснено определенной выборкой пациентов (госпитализированные в стационар) и их возрастом (в среднем 74,9 7,7 года). Известно, что падение - это гериатрический синдром, возникающий в результате суммарного эффекта действия различных внешних и внутренних факторов. Ключевые стратегии профилактики падений включают выявление потенциально модифицируемых факторов риска, таких как проблемы со зрением, ортостатическая гипотензия, полипрагмазия, прием неподходящих лекарственных препаратов, и снижение риска падений путем изменения этих факторов риска [11].

Одним из распространенных факторов риска падений у пожилых людей является полипрагмазия. Распространенность полипрагмазии, по данным австралийских ученых, составляет 76\% [13]. Исследователи из Турции показали, что среди 1579 пожилых пациентов полипрагмазия была задокументирована у 56,7\%, а среднее коли-

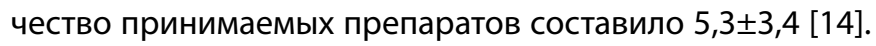
В нашем исследовании полипрагмазия была определена 
Таблица 2. Структура лекарственной терапии до госпитализации

\begin{tabular}{|c|c|c|c|c|}
\hline Группа лекарственных препаратов & $\begin{array}{c}\text { Пациенты } \\
\text { с падениями } \\
(\mathrm{n}=332)\end{array}$ & $\%$ & $\begin{array}{c}\text { Пациенты без } \\
\text { падений } \\
(n=323)\end{array}$ & $\%$ \\
\hline иАПФ & 147 & 44,3 & 157 & 48,6 \\
\hline Сартаны & 99 & 29,8 & 87 & 26,9 \\
\hline Бета-блокаторы & 158 & 47,6 & 160 & 49,5 \\
\hline БКК & 98 & 29,5 & 100 & 30,9 \\
\hline Диуретики & 109 & 32,8 & 98 & 30,3 \\
\hline Альфа-блокаторы & 17 & 5,1 & 11 & 3,4 \\
\hline Гипотензивные препараты центрального действия (моксонидин) & 8 & 2,4 & 9 & 2,8 \\
\hline Статины & 102 & 30,7 & 119 & 36,8 \\
\hline Антиагреганты & 154 & 46,4 & 149 & 46,1 \\
\hline Антикоагулянты & 31 & 9,3 & 32 & 9,9 \\
\hline Антиаритмические препараты & 14 & 4,2 & 13 & 4 \\
\hline Дигоксин & 7 & 2,1 & 7 & 2,2 \\
\hline Гипогликемические пероральные препараты & 68 & 20,5 & 61 & 18,9 \\
\hline Инсулин & 6 & 1,8 & 5 & 1,5 \\
\hline Противодементные препараты & 6 & 1,8 & 6 & 1,9 \\
\hline Снотворные препараты & 26 & 7,8 & 13 & $4^{*}$ \\
\hline Противопаркинсонические препараты & 13 & 3,9 & 8 & 2,5 \\
\hline Ноотропы & 31 & 9,3 & 22 & 6,9 \\
\hline Антиконвульсанты & 4 & 1,2 & 4 & 1,2 \\
\hline Антидепрессанты & 6 & 1,8 & 8 & 2,5 \\
\hline Бетагистин & 9 & 2,7 & 7 & 2,2 \\
\hline НПВП & 67 & 20,2 & 34 & $10,5^{*}$ \\
\hline XC, гС & 6 & 1,8 & 4 & 1,2 \\
\hline Гипоурикемические препараты & 9 & 2,7 & 7 & 2,2 \\
\hline ИПп & 45 & 13,6 & 32 & 9,9 \\
\hline Препараты кальция & 18 & 5,4 & 25 & 7,7 \\
\hline Антирезорбтивные препараты & 5 & 1,5 & 4 & 1,2 \\
\hline Витамин D & 18 & 5,4 & 29 & 8,9 \\
\hline Гормоны щитовидной железы & 37 & 11,1 & 23 & 7,1 \\
\hline Ингаляционные бета-адреномиметики & 7 & 2,1 & 6 & 1,9 \\
\hline Ингаляционные ГКС & 6 & 1,8 & 3 & 0,9 \\
\hline Ферменты & 12 & 3,6 & 12 & 3,8 \\
\hline Слабительные & 22 & 6,6 & 24 & 7,4 \\
\hline Препараты железа & 1 & 0,3 & 1 & 0,3 \\
\hline Фолиевая кислота & 0 & 0 & 1 & 0,3 \\
\hline Венотоники & 6 & 1,8 & 6 & 1,9 \\
\hline Валидол, корвалол & 6 & 1,8 & 5 & 1,5 \\
\hline
\end{tabular}

Примечание: иАПФ - ингибиторы ангиотензинпревращающего фермента; БКК - блокаторы кальциевых каналов; НПВП нестероидные противовоспалительные препараты; ХС - хондроитин сульфат; ГС — глюкозамин сульфат; ИПП - ингибиторы протонной помпы; ГКС - глюкокортикостероиды; *p<0,05. 
Таблица 3. Структура лекарственной терапии до и после выписки из стационара у пациентов с синдромом падений

\begin{tabular}{|c|c|c|c|c|}
\hline Группа лекарственных препаратов & $\begin{array}{c}\text { До } \\
\text { госпитализации } \\
\text { (n) }\end{array}$ & $\%$ & $\begin{array}{c}\text { После } \\
\text { выписки } \\
\text { (n) }\end{array}$ & $\%$ \\
\hline иАПФ & 147 & 44,3 & 131 & 39,5 \\
\hline Сартаны & 99 & 29,8 & 111 & 33,4 \\
\hline Бета-блокаторы & 158 & 47,6 & 155 & 46,7 \\
\hline БКK & 98 & 29,5 & 96 & 28,9 \\
\hline Диуретики & 109 & 32,8 & 90 & 27,1 \\
\hline Альфа-блокаторы & 17 & 5,1 & 11 & 3,3 \\
\hline Гипотензивные препараты центрального действия (моксонидин) & 8 & 2,4 & 5 & 1,5 \\
\hline Статины & 102 & 30,7 & 181 & $54,5^{*}$ \\
\hline Антиагреганты & 154 & 46,4 & 102 & $30,7^{*}$ \\
\hline Антикоагулянты & 31 & 9,3 & 40 & 12 \\
\hline Антиаритмические препараты & 14 & 4,2 & 14 & 4,2 \\
\hline Дигоксин & 7 & 2,1 & 2 & $0,6^{*}$ \\
\hline Гипогликемические пероральные препараты & 68 & 20,5 & 57 & 17,2 \\
\hline Инсулин & 6 & 1,8 & 14 & 4,2 \\
\hline Противодементные препараты & 6 & 1,8 & 19 & $5,7^{*}$ \\
\hline Снотворные препараты & 26 & 7,8 & 6 & $1,8^{*}$ \\
\hline Противопаркинсонические препараты & 13 & 3,9 & 13 & 3,9 \\
\hline Ноотропы & 31 & 9,3 & 4 & $1,2^{*}$ \\
\hline Антиконвульсанты & 4 & 1,2 & 12 & $3,6^{*}$ \\
\hline Антидепрессанты & 6 & 1,8 & 20 & $6^{*}$ \\
\hline Бетагистин & 9 & 2,7 & 12 & 3,6 \\
\hline НПВП & 67 & 20,2 & 14 & $4,2^{*}$ \\
\hline $\mathrm{XC}$, гC & 6 & 1,8 & 15 & $4,5^{*}$ \\
\hline Гипоурикемические препараты & 9 & 2,7 & 9 & 2,7 \\
\hline ипп & 45 & 13,6 & 52 & 15,7 \\
\hline Препараты кальция & 18 & 5,4 & 44 & $13,3^{*}$ \\
\hline Антирезорбтивные препараты & 5 & 1,5 & 29 & $8,7^{*}$ \\
\hline Витамин B & 18 & 5,4 & 332 & $100^{*}$ \\
\hline Гормоны щитовидной железы & 37 & 11,1 & 39 & 11,7 \\
\hline Ингаляционные бета-адреномиметики & 7 & 2,1 & 9 & 2,7 \\
\hline Ингаляционные ГКС & 6 & 1,8 & 7 & 2,1 \\
\hline Ферменты & 12 & 3,6 & 5 & 1,5 \\
\hline Слабительные препараты & 22 & 6,6 & 9 & 2,7 \\
\hline Препараты железа & 1 & 0,3 & 16 & $4,8^{*}$ \\
\hline Фолиевая кислота & 0 & 0 & 8 & $2,4^{*}$ \\
\hline Венотоники & 6 & 1,8 & 6 & 1,8 \\
\hline Валидол, корвалол & 6 & 1,8 & 0 & $0^{*}$ \\
\hline
\end{tabular}

Примечание: иАПФ - ингибиторы ангиотензинпревращающего фермента; БКК - блокаторы кальциевых каналов; НПВП нестероидные противовоспалительные препараты; ХС - хондроитин сульфат; ГС — глюкозамин сульфат; ИПП - ингибиторы протонной помпы; ГКС - глюкокортикостероиды; * $\mathrm{p}<0,05$. 
Таблица 4. STOPР-критерии и их частота у пациентов с падениями

\begin{tabular}{|c|c|c|}
\hline Критерий & $\mathbf{n}$ & $\begin{array}{c}\text { \% всех } \\
\text { STOPP-критериев }\end{array}$ \\
\hline $\begin{array}{l}\text { НПВП при хронической почечной недостаточности: СКФ 20-50 мл/мин } \\
\text { (риск ухудшения почечной функции) }\end{array}$ & 5 & 3,5 \\
\hline $\begin{array}{l}\text { Длительный прием НПВП (более } 3 \text { мес) для облегчения умеренной боли } \\
\text { при остеоартрите }\end{array}$ & 29 & 20,6 \\
\hline $\begin{array}{l}\text { НПВП с антагонистом витамина К, прямым ингибитором тромбина или } \\
\text { ингибитором фактора Ха (увеличение риска желудочно-кишечного кровотечения) }\end{array}$ & 7 & 5 \\
\hline $\begin{array}{l}\text { НПВП с антиагрегантами без профилактики ингибитором протонной помпы } \\
\text { (повышенный риск язвенной болезни) }\end{array}$ & 12 & 8,5 \\
\hline $\begin{array}{l}\text { Метформин у пациентов с СКФ <30 мл/мин/1,73 м² (риск развития } \\
\text { молочнокислого ацидоза) }\end{array}$ & 3 & 2,1 \\
\hline $\begin{array}{l}\text { Препараты сульфонилмочевины длительного действия (глибенкламид, } \\
\text { хлорпропамид, глимепирид) при сахарном диабете } 2 \text { типа (повышается риск } \\
\text { длительной гипогликемии) }\end{array}$ & 8 & 5,8 \\
\hline $\begin{array}{l}\text { Дигоксин для лечения сердечной недостаточности с сохранной систолической } \\
\text { функцией (нет доказательств пользы) }\end{array}$ & 5 & 3,5 \\
\hline $\begin{array}{l}\text { Гипотензивные препараты центрального действия (метилдопа, клонидин, } \\
\text { моксонидин, рилменидин, гуанфацин) за исключением тех случаев, когда отмечается } \\
\text { непереносимость или недостаточная эффективность антигипертензивных } \\
\text { препаратов других классов (антигипертензивные препараты центрального действия } \\
\text { переносятся пожилыми людьми в целом хуже, чем молодыми пациентами) }\end{array}$ & 3 & 2,1 \\
\hline $\begin{array}{l}\text { Бета-адреноблокаторы при сахарном диабете } 2 \text { типа и частых эпизодах } \\
\text { гипогликемии, т.е. более } 1 \text { эпизода в месяц (риск маскировки гипогликемических } \\
\text { симптомов) }\end{array}$ & 5 & 3,5 \\
\hline Блокаторы кальциевых каналов при хроническом запоре (могут усилить запор) & 2 & 1,4 \\
\hline $\begin{array}{l}\text { Ацетилсалициловая кислота при отсутствии признаков поражения коронарных, } \\
\text { мозговых или периферических артерий или атеротромботического события }\end{array}$ & 62 & 44 \\
\hline
\end{tabular}

Примечание: НПВП — нестероидные противовоспалительные препараты; СКФ — скорость клубочковой фильтрации.

Таблица 5. START-критерии и их частота у пациентов с падениями

\begin{tabular}{|c|c|c|}
\hline Характеристика & (n) & $\begin{array}{l}\text { \% всех } \\
\text { START-ритериев }\end{array}$ \\
\hline $\begin{array}{l}\text { Кальций и витамин D у пациентов с остеопорозом (доказанным } \\
\text { инструментальными методами, а также при наличии остеопоротического } \\
\text { перелома в анамнезе или приобретенного дорсального кифоза) }\end{array}$ & 26 & 5,7 \\
\hline $\begin{array}{l}\text { Препараты, предотвращающие резорбцию костной ткани, и анаболические } \\
\text { стероиды (бисфосфонаты, терипаратид, стронция ранелат, деносумаб) } \\
\text { у пациентов с остеопорозом, если нет противопоказаний или в анамнезе имеются } \\
\text { остеопоротические переломы }\end{array}$ & 24 & 5,2 \\
\hline $\begin{array}{l}\text { Витамин D у пожилых пациентов с остеопенией или с падениями в анамнезе, } \\
\text { которые выходят из дома }\end{array}$ & 314 & 68,6 \\
\hline $\begin{array}{l}\text { Ацетилсалициловая кислота при коронарной болезни сердца в анамнезе } \\
\text { у пациентов с синусовым ритмом }\end{array}$ & 10 & 2,2 \\
\hline $\begin{array}{l}\text { Статины при поражении коронарных, мозговых или периферических артерий } \\
\text { в анамнезе при ожидаемой продолжительности жизни более } 5 \text { лет }\end{array}$ & 79 & 17,2 \\
\hline Бета-адреноблокаторы при стабильной стенокардии & 2 & 0,4 \\
\hline $\begin{array}{l}\text { Ингибиторы ацетилхолинэстеразы (донепезил, ривастигмин, галантамин) для } \\
\text { лечения легкой и среднетяжелой болезни Альцгеймера и деменции при болезни } \\
\text { телец Леви (ривастигмин) }\end{array}$ & 3 & 0,7 \\
\hline
\end{tabular}


у 43,9\% пациентов с падениями и у 37,8\% пациентов без падений, а среднее количество лекарственных препара-

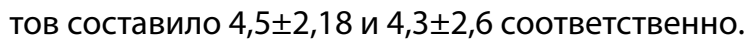

Некоторые исследования не показали ассоциаций между полипрагмазией и падениями $[15,16]$. Однако большинство исследований демонстрируют, что прием 4 и более лекарственных препаратов связан с увеличением частоты падений и случаев повторных падений $[4,11,17,18]$. Кроме того, предыдущие исследования показывают, что конкретные классы лекарств (например, гипотензивные средства, анальгетики, седативные и снотворные средства, антидепрессанты, антихолинергические препараты) увеличивают риск падений и травм, связанных с падением [19, 20]. Мы получили данные, свидетельствующие об ассоциации падений с приемом НПВП и снотворных препаратов.

Применяя STOPP/START-критерии, у пациентов с падениями была оптимизирована лекарственная терапия. Этим пациентам к терапии чаще были добавлены статины, противодементные препараты, антиконвульсанты и антидепрессанты, препараты хондроитина сульфата и глюкозамина сульфата, препараты кальция и антирезорбтивная терапия, противоанемические препараты. Особо необходимо подчеркнуть, что самым частым назначением был витамин D. Аналогичные данные по группам лекарственных препаратов приведены и в зарубежном исследовании [14]. Группами лекарств, которые чаще отменялись после оценки фармакотерапии, оказались антиагреганты, дигоксин, снотворные препараты, ноотропы, НПВП. А препараты валидол и корвалол были отменены у всех пациентов, их принимавших.

\section{Клиническая значимость результатов}

Полученные данные продемонстрировали ассоциацию падений с приемом НПВП и снотворных препаратов, что требует особого внимания клиницистов при изучении лекарственного анамнеза пациентов с падениями. Наше исследование подтвердило необходимость тщательной и регулярной ревизии лекарственных препаратов с использованием STOPP/START-критериев у лиц пожилого возраста. Это позволяет снизить распространенность полипрагмазии, изменить качественный состав лекарственного перечня пациента, планировать долговременную лекарственную терапию, что приведет к снижению риска и частоты падений и ассоциированных с ними осложнений.

\section{Ограничения исследования}

Оценка фармакотерапии проводилась у пациентов, госпитализированных в гериатрическое отделение.
Оценить фармакотерапию, которую получают пациенты, наблюдающиеся гериатром на амбулаторном этапе или проживающие в домах престарелых, возможности не было.

\section{Направления дальнейших исследований}

Считаем целесообразным проведение дальнейших исследований по изучению распространенности полипрагмазии, фармакотерапии в разных популяциях пожилых пациентов с целью обоснованной коррекции и снижения гериатрических рисков.

\section{ЗАКЛЮЧЕНИЕ}

Пожилые пациенты с синдромом падений чаще принимали снотворные препараты и НПВП. При аудите догоспитальной лекарственной терапии STOPP-критерии наиболее часто касались назначения НПВП и ацетилсалициловой кислоты, а START-критерии выявили отсутствие назначения витамина D и статинов.

\section{ДОПОЛНИТЕЛЬНАЯ ИНФОРМАЦИЯ}

Источники финансирования. Исследование было выполнено в рамках Государственного задания «Оптимизация диагностики и ведения сочетанных заболеваний опорно-двигательного аппарата у пациентов пожилого и старческого возраста с целью сохранения автономности».

Конфликт интересов. Авторы декларируют отсутствие явных и потенциальных конфликтов интересов, связанных с содержанием настоящей статьи.

Участие авторов. Ховасова Н.О. - существенный вклад в концепцию и дизайн исследования, в получение, анализ данных или интерпретацию результатов, написание статьи; Наумов А.В. - существенный вклад в концепцию и дизайн исследования, в получение, анализ данных или интерпретацию результатов, написание статьи; Ткачева О.Н. - существенный вклад в получение, анализ данных или интерпретацию результатов, внесение в рукопись существенной правки с целью повышения научной ценности статьи; Дудинская Е.Н. - существенный вклад в получение, анализ данных или интерпретацию результатов, внесение в рукопись существенной правки с целью повышения научной ценности статьи; Мороз В.И. существенный вклад в получение, анализ данных или интерпретацию результатов, написание статьи.

Все авторы одобрили финальную версию статьи перед публикацией, выразили согласие нести ответственность за все аспекты работы, подразумевающую надлежащее изучение и решение вопросов, связанных с точностью или добросовестностью любой части работы.

\section{СПИСОК ЛИТЕРАТУРЫ | REFERENCES}

1. Клинические рекомендации «Падения у пациентов пожилого и старческого возраста», 2020. [Интернет]. Доступно по ссылке: http://cr.rosminzdrav.ru/recomend/600_2 [Klinicheskie rekomendatsii «Padeniya u patsientov pozhilogo i starcheskogo vozrasta», 2020. (In Russ.). [Internet]. Available from: http://cr.rosminzdrav.ru/recomend/600_2

2. Jokanovic N, Tan ECK, Dooley MJ, et al. Prevalence and Factors Associated With Polypharmacy in Long-Term Care Facilities: A Systematic Review. J Am Med Dir Assoc. 2015;16(6):535.e1-535.e12. doi: https://doi.org/10.1016/j.jamda.2015.03.003
3. Ткачева О.Н., Котовская Ю.В., Рунихина Н.К., и др. Клинические рекомендации «Старческая астения» // Российский журнал гериатрической медичины. — 2020. — №1. — С. 11-46. [Tkacheva ON, Kotovskaya YV, Runikhina NK, et al. Clinical guidelines on frailty. Russ J Geriatr Med. 2020;128(1):11-46. (In Russ.)]. doi: https://doi.org/10.37586/2686-8636-1-2020-11-46

4. Dhalwani NN, Fahami R, Sathanapally H, et al. Association between polypharmacy and falls in older adults: a longitudinal study from England. BMJ Open. 2017;7(10):e016358. doi: https://doi.org/10.1136/bmjopen-2017-016358 
5. Maher RL, Hanlon J, Hajjar ER. Clinical consequences of polypharmacy in elderly. Expert Opin Drug Saf. 2014;13(1):57-65 doi: https://doi.org/10.1517/14740338.2013.827660

6. Hein C, Forgues A, Piau A, Sommet A, et al. Impact of polypharmacy on occurrence of delirium in elderly emergency patients. J Am Med Dir Assoc. 2014;15(11):850.e1 1-15. doi: https://doi.org/10.1016/j.jamda.2014.08.012

7. Lapeyre-Mestre M, de Castro AMR, Bareille M-P, et al. Nonsteroidal anti-inflammatory drug-related hepatic damage in France and Spain: analysis from national spontaneous reporting systems. Fundam Clin Pharmacol. 2006;20(4):391-395. doi: https://doi.org/10.1111/j.1472-8206.2006.00416.x

8. Leelakanok N, Holcombe AL, Lund BC, et al. Association between polypharmacy and death: A systematic review and meta-analysis. J Am Pharm Assoc. 2017;57(6):729-738. doi: https://doi.org/10.1016/j.japh.2017.06.002

9. O'Mahony D, O'Sullivan D, Byrne S, et al. STOPP/ START criteria for potentially inappropriate prescribing in older people: version 2. Age Ageing. 2014;44(2):213-218 doi: https://doi.org/10.1093/ageing/afu145

10. O'Mahony D. STOPP/START criteria for potentially inappropriate medications/potential prescribing omissions in older people: origin and progress. Expert Rev Clin Pharmacol. 2020;13(1):15-22. doi: https://doi.org/10.1080/17512433.2020.1697676

11. Zia A, Kamaruzzaman SB, Tan MP. Polypharmacy and falls in older people: Balancing evidence-based medicine against falls risk. Postgrad Med. 2015;127(3):330-337. doi: https://doi.org/10.1080/00325481.2014.996112

12. von Heideken Wågert $P$, Gustafson $Y$, Kallin $K$, et al. Falls in very old people: The population-based Umeå 85+ Study in Sweden. Arch Gerontol Geriatr. 2009;49(3):390-396. doi: https://doi.org/10.1016/j.archger.2008.12.005
13. Hubbard RE, Peel NM, Scott IA, et al. Polypharmacy among inpatients aged 70 years or older in Australia. Med J Aust. 2015;202(7):373-377. doi: https://doi.org/10.5694/mja13.00172

14. Unutmaz GD, Soysal P, Tuven B, Isik AT. Costs of medication in older patients: Before and after comprehensive geriatric assessment. Clin Interv Aging. 2018 doi: https://doi.org/10.2147/CIA.S159966

15. Lawlor DA, Patel R, Ebrahim S. Association between falls in elderly women and chronic diseases and drug use: cross sectional study. BMJ. 2003;327(7417):712-717. doi: https://doi.org/10.1136/bmj.327.7417.712

16. Zia A, Kamaruzzaman SB, Tan MP. The consumption of two or more fall risk-increasing drugs rather than polypharmacy is associated with falls. Geriatr Gerontol Int. 2017;17(3):463-470. doi: https://doi.org/10.1111/ggi.12741

17. Wong $\mathrm{H}$, Heuberger $\mathrm{R}$, Logomarsino J, Hewlings $\mathrm{S}$. Associations between alcohol use, polypharmacy and falls in older adults. Nurs Older People. 2016;28(1):30-36. doi: https://doi.org/10.7748/nop.28.1.30.s22

18. Helgadóttir B, Laflamme L, Monárrez-Espino J, Möller J. Medication and fall injury in the elderly population; do individual demographics, health status and lifestyle matter? BMC Geriatr. 2014;14(1):92. doi: https://doi.org/10.1186/1471-2318-14-92

19. Tinetti ME, Han L, Lee DSH, et al. Antihypertensive Medications and Serious Fall Injuries in a Nationally Representative Sample of Older Adults. JAMA Intern Med. 2014;174(4):588. doi: https://doi.org/10.1001/jamainternmed.2013.14764

20. Johnell K, Jonasdottir Bergman G, Fastbom J, et al. Psychotropic drugs and the risk of fall injuries, hospitalisations and mortality among older adults. Int J Geriatr Psychiatry. 2017;32(4):414-420. doi: https://doi.org/10.1002/gps.4483

\section{ИНФОРМАЦИЯ ОБ АВТОРАХ [AUTHORS INFO]}

*Ховасова Наталья Олеговна, К.М.Н., доцент [Natalia O. Khovasova, PhD, associate Professor]; адрес: г. Москва, ул. 1-я Леонова, д. 16 [Address: Moscow, First Leonova str., 16]; e-library SPIN: 7387-7710; ORCID: 0000-0002-3066-4866; e-mail: natashahov@mail.ru

Наумов Антон Вячеславович, д.М.Н., доцент, професcop [Anton V. Naumov, PhD, Professor]; e-library SPIN: 4763-9738; ORCID: 0000-0002-6253-621X; e-mail: nanton78@gmail.com

Ткачева Ольга Николаевна, д.м.Н., профессор [Olga N. Tkacheva, PhD, Professor]; e-library SPIN: 6129-5809;

ORCID: 0000-0002-4193-688X; e-mail: ton@rgnkc.ru

Дудинская Екатерина Наильевна, К.м.н. [Ekaterina N. Dudinskaya, PhD]; e-library SPIN: 4985-6315;

ORCID: 0000-0001-7891-6850; e-mail: katharina.gin@gmail.com

Мороз Виктория Ивановна [Victoriya I. Moroz]; e-library SPIN: 9739-6940; ORCID: 0000-0001-6040-1090;

e-mail: vikulya-moroz@yandex.ru

\section{ИНФОРМАЦИЯ}

Рукопись получена: 10.03.2021. Одобрена к публикации: 27.05.2021.

\section{ЦИТИРОВАТЬ:}

Ховасова Н.О., Наумов А.В., Ткачева О.Н., Дудинская Е.Н., Мороз В.И. Полипрагмазия у пожилых пациентов с синдромом падений // Остеопороз и остеоnатиu. — 2021. - Т. 24. — №1. — C. 10-18. doi: https://doi.org/10.14341/osteo12716

\section{TO CITE THIS ARTICLE:}

Khovasova NO, Naumov AV, Tkacheva ON, Dudinskaya EN, Moroz VI. Polypharmacy in elderly patients with falls. Osteoporosis and bone diseases. 2021;24(1):10-18. doi: https://doi.org/10.14341/osteo12719 